\title{
The association between hyperuricemia and left atrial enlargement in healthy adults
}

\author{
Danhong Fang ${ }^{1,2,3}$, Na Wang ${ }^{3}$, Qinfen Chen ${ }^{3}$, Gaojun $\mathrm{Wu}^{2}$, Jiansheng $\mathrm{Wu}^{3}$, Wenbin Zhang ${ }^{1}$, Guosheng Fu ${ }^{1}$ \\ ${ }^{1}$ Department of Cardiology, Sir Run Run Shaw Hospital, Zhejiang University School of Medicine, Hangzhou, China; ${ }^{2}$ Department of Cardiology, \\ The First Affiliated Hospital of Wenzhou Medical University, Wenzhou, China; ${ }^{3}$ Department of Medical and health care center, The First Affiliated \\ Hospital of Wenzhou Medical University, Wenzhou, China \\ Contributions: (I) Conception and design: G Fu, D Fang, G Wu; (II) Administrative support: G Fu, G Wu; (III) Provision of study materials or \\ patients: J Wu; (IV) Collection and assembly of data: N Wang, Q Chen; (V) Data analysis and interpretation: D Fang, W Zhang; (VI) Manuscript \\ writing: All authors; (VII) Final approval of manuscript: All authors. \\ Correspondence to: Guosheng Fu. Department of Cardiology, Sir Run Run Shaw Hospital, Zhejiang University School of Medicine, Hangzhou \\ 310016, China. Email: fugs@zju.edu.cn
}

\begin{abstract}
Background: Although hyperuricemia (HU) has been reported to be related to atrial fibrillation (AF), the relationship between HU and left atrial (LA) enlargement is unclear. The aim of this study was to investigate the interaction between HU and LA enlargement in healthy adults in China.

Methods: This study retrospectively surveyed 5,392 people (3,336 males and 2,056 females) who underwent health checks. Basic data were obtained from all participants, including baseline characteristics and general health status through laboratory tests, echocardiography, and interviews. Multinomial logistic regression was used to analyze the experimental data and determine the association between HU and LA enlargement. In addition, the relationship between HU and LA enlargement in different gender groups was analyzed.

Results: The prevalence of HU in this study was $20.3 \%$. Compared with the normal LA group, the prevalence of $\mathrm{HU}$ in the LA enlargement group was significantly higher $[31.5 \%$ vs. $18.1 \%$; $\mathrm{P}<0.001$; odds ratio $(\mathrm{OR})=2.09,95 \%$ confidence interval $(\mathrm{CI}): 1.78-2.45]$. After adjustment for confounding variables, the interrelation of $\mathrm{HU}$ on LA enlargement was found to be independent in the total participants $(\mathrm{OR}=1.25$, 95\% CI: $1.04-1.51 ; \mathrm{P}=0.017)$, especially in women ( $\mathrm{OR}=1.73 ; 95 \% \mathrm{CI}: 1.10-2.74 ; \mathrm{P}=0.019)$ but not in men $(\mathrm{P}=0.195)$.
\end{abstract}

Conclusions: HU is independently associated with LA enlargement in healthy adults, especially in women.

Keywords: Hyperuricemia (HU); uric acid; left atrium; atrial remodeling

Submitted Apr 30, 2021. Accepted for publication Jul 15, 2021.

doi: $10.21037 /$ atm-21-3402

View this article at: https://dx.doi.org/10.21037/atm-21-3402

\section{Introduction}

One of the most common clinical types of arrhythmia is atrial fibrillation (AF). Patients with AF are 5 times more likely to have a stroke, and AF approximately doubles the risk of death (1) and can also cause heart failure. In addition, patients with hyperuricemia (HU) are more likely to develop paroxysmal or persistent $\mathrm{AF}$ and have an increased risk of AF after cardiovascular surgery. The reason for this phenomenon may be based on the inflammation or oxidative stress of the disease (2).
The most important aspects in the process of AF are electrical remodeling and structural remodeling, with inflammation and oxidative stress also being significant factors (3-6). However, structural remodeling may aggravate the extent of left atrial (LA) enlargement, which is critical in the progression of AF (7). Meanwhile, several researchers have reported high serum uric acid (SUA) level to be significantly correlated with enlarged LA size and HU to be associated with a larger LA diameter $(8,9)$.

In order to examine the development of $\mathrm{HU}$ and $\mathrm{AF}$, it 
is essential to explore the atrial remodeling caused by HU. However, previous experimental studies have not confirmed the connection between HU and LA enlargement in large sample size. Thus, this study aimed to understand the interaction between $\mathrm{HU}$ and LA enlargement in a relatively large healthy adults in China and analyze any potential sex differences.

We present the following article in accordance with the STROBE reporting checklist (available at https://dx.doi. org/10.21037/atm-21-3402).

\section{Methods}

\section{Study population}

All subjects underwent routine examinations and echocardiography in the First Affiliated Hospital of Wenzhou Medical University from January 2015 to December 2017. The patients' general physical condition, height, weight and body mass index (BMI), blood pressure (BP), past history, and drug use history were recorded. According to expert consensus and standard on weight management for overweight or obese status in China, a BMI greater or equal to $24 \mathrm{~kg} / \mathrm{m}^{2}$ and less than $28 \mathrm{~kg} / \mathrm{m}^{2}$ is considered overweight, while a BMI greater or equal to $28 \mathrm{~kg} / \mathrm{m}^{2}$ is considered obese. A total of 6,909 participants were screened, with 1,517 being excluded based on the following exclusion criteria: (I) cardiovascular disease type, including congestive heart failure, congenital heart diseases, cardiomyopathy, coronary heart disease, and AF; (II) anemia; (III) thyroid dysfunction; (IV) history of malignant tumor; (V) history of rheumatic immune system disease; and (VI) ingestion of diuretics or other drugs that can increase SUA. Finally, 5,392 subjects in this experiment (3,336 males and 2,056 females) were included. The participants in this study were divided into an LA enlargement group and a normal LA group. All procedures performed in this study involving human participants were in accordance with the Declaration of Helsinki (as revised in 2013). The study was approved by Institutional Review Board (IRB) of the first affiliated hospital of Wenzhou Medical University (No. 2020107). Individual consent for this retrospective analysis was waived.

\section{Data collection and diagnostic definition}

After patients fasted for 8 hours, blood samples were collected from the median cubital vein and analyzed. A automatic biochemical analyzer (Beckman Coulter AU5800, USA) was then used to analyze biochemical indicators such as SUA, total cholesterol (TC), triglyceride (TG), highdensity lipoprotein cholesterol (HDL-C), low-density lipoprotein cholesterol (LDL-C), fasting plasma glucose (FPG) and serum creatinine (SCr). Definition of $\mathrm{HU}$ : in a normal purine diet, fasting SUA level is higher than $420 \mu \mathrm{mol} / \mathrm{L}$ in men and higher than $360 \mu \mathrm{mol} / \mathrm{L}$ in women, or taking xanthine oxidase inhibitors (10). BP measurements were taken according to Hypertension Canada's 2016 guidelines (11). BP was measured by a trained medical staff using auscultatory or automatic electronic sphygmomanometers (HEM-705CP, Omron, Kyoto, Japan). An ultrasound system (Vivid E95, GE Healthcare, Amersham, UK) was used for echocardiography. Degree of LA enlargement and left ventricular ejection fraction were determined from $\mathrm{M}$-mode images following a standardized imaging protocol. LA enlargement was considered to be LA diameter $>40 \mathrm{~mm}$, and normal LA was considered to be LA diameter $\leq 40 \mathrm{~mm}(12)$.

\section{Statistical analysis}

SPSS 19.0 software (IBM Corporation, Armonk, NY, USA) was used for data analysis. Measurement data are expressed as mean \pm standard deviation and were compared with $t$ test or one-way analysis of variance (ANOVA). If the variable was normally distributed, a $\chi^{2}$ test or Fisher's exact test was used; otherwise, the Mann-Whitney $\mathrm{U}$ test was performed. The associations between HU and LA enlargement were analyzed through multiple logistic regression. The results are reported with odds ratios (ORs) and $95 \%$ confidence intervals (CIs). A $\mathrm{P}$ value $<0.05$ indicated that the difference in component data was statistically significant.

\section{Results}

\section{Participant characteristics}

Table 1 contains the general demographic and clinical information of the participants. Table 2 shows the comparison of demographic and clinical information between men and women. Out of the 5,392 subjects, 891 (16.5\%) were diagnosed with LA enlargement. There were significant differences between the LA enlargement group and normal LA group in age, prevalence of HU, BMI, systolic blood pressure (SBP), diastolic blood pressure (DBP), SUA, TG, HDL-C, FPG, SCr, and history of 
Table 1 Demographic and clinical characteristics of the LA enlargement group and normal LA group

\begin{tabular}{|c|c|c|c|}
\hline Variables & LA enlargement $(n=891)$ & Normal LA $(n=4,501)$ & $P$ value \\
\hline Hyperuricemia, n (\%) & $281(31.5)$ & $813(18.1)$ & $<0.001$ \\
\hline Age (years) & $53.81 \pm 11.06$ & $46.95 \pm 11.03$ & $<0.001$ \\
\hline $\operatorname{BMl}\left(\mathrm{kg} / \mathrm{m}^{2}\right)$ & $26.77 \pm 2.84$ & $23.42 \pm 3.34$ & $<0.001$ \\
\hline $\mathrm{DBP}(\mathrm{mmHg})$ & $79.73 \pm 12.32$ & $73.61 \pm 12.52$ & $<0.001$ \\
\hline SUA $(\mu \mathrm{mol} / \mathrm{L})$ & $374.62 \pm 85.73$ & $333.14 \pm 88.74$ & $<0.001$ \\
\hline $\mathrm{TC}(\mathrm{mmol} / \mathrm{L})$ & $5.33 \pm 1.03$ & $5.31 \pm 1.11$ & 0.619 \\
\hline $\mathrm{TG}(\mathrm{mmol} / \mathrm{L})$ & $2.21 \pm 1.73$ & $1.77 \pm 1.63$ & $<0.001$ \\
\hline FPG (mmol/L) & $5.35 \pm 1.58$ & $4.91 \pm 1.24$ & $<0.001$ \\
\hline Creatinine $(\mu \mathrm{mol} / \mathrm{L})$ & $69.50 \pm 15.30$ & $65.15 \pm 14.23$ & $<0.001$ \\
\hline \multicolumn{4}{|l|}{ Medical history, n (\%) } \\
\hline Hypertension & $142(15.9)$ & $361(8.0)$ & $<0.001$ \\
\hline Diabetes mellitus & $47(5.3)$ & $92(2.0)$ & $<0.001$ \\
\hline
\end{tabular}

LA, left atrium; BMI, body mass index; SBP, systolic blood pressure; DBP, diastolic blood pressure; SUA, serum uric acid; TC, total cholesterol; TG, triglyceride; HDL-C, high-density lipoprotein cholesterol; LDL-C, low-density lipoprotein cholesterol; FPG, fasting plasma glucose.

hypertension and diabetes $(\mathrm{P}<0.001)$. However, there were no differences in the TC and LDL-C between the control group and the study group.

\section{$H U$ was related to the incidence of $L A$ enlargement}

In this study, patients with $\mathrm{HU}$ accounted for $20.3 \%$ of the total sample size, and the prevalence of $\mathrm{HU}$ in LA enlargement group was significantly higher than in normal LA group (31.5\% vs. $18.1 \%$; $\mathrm{P}<0.001$; Table 1). In univariate analysis, the experimental results showed that subjects with $\mathrm{HU}$ had an increased incidence of LA enlargement (OR $=2.09,95 \%$ CI: $1.78-2.45 ; \mathrm{P}<0.001)$ compared with subjects without $\mathrm{HU}$ (Table 3). Other risk factors, such as gender, age, BMI, TG, HDL-C, FPG, and SCr were significantly associated with LA enlargement $(\mathrm{P}<0.001)$. No correlation was found between TC, LDL-C and LA expansion. After adjustments for the above clinical and biochemical covariates were made, correlation was still found between $\mathrm{HU}$ and LA enlargement in the total sample size (OR $=1.25,95 \%$ CI: 1.04-1.51; $\mathrm{P}=0.017$; Table 3).

\section{The association between $H U$ and $L A$ enlargement in gender subgroup analysis}

Table 4 and Table 5 show the association between HU and LA enlargement in the gender subgroup analysis. In the univariate analysis, $\mathrm{HU}$ was significantly related to LA enlargement in women $(\mathrm{OR}=3.53,95 \% \mathrm{CI}$ : 2.35-5.26; $\mathrm{P}<0.001)$ and in men ( $\mathrm{OR}=1.48$, 95\% CI: $1.24-1.77$; $\mathrm{P}<0.001)$, respectively. Other risk factors, such as age, BMI, SBP, DBP, TG, HDL-C and FPG, were significantly associated with LA enlargement in both men and women $(\mathrm{P}<0.05)$. LDL-C was associated with LA enlargement in women $(\mathrm{P}=0.027)$. After adjustment for the above confounding factors, $\mathrm{HU}$ and LA expansion still remained independent in women $(\mathrm{OR}=1.73,95 \% \mathrm{CI}$ : $1.10-2.74$; $\mathrm{P}=0.019$ ), but this relationship was not found in men (OR $=1.14,95 \%$ CI: $0.93-1.40 ; \mathrm{P}=0.195$ ).

\section{Discussion}

Our findings suggest that HU is independently associated 
Table 2 Demographic and clinical characteristics of the LA enlargement group and the normal LA group by gender

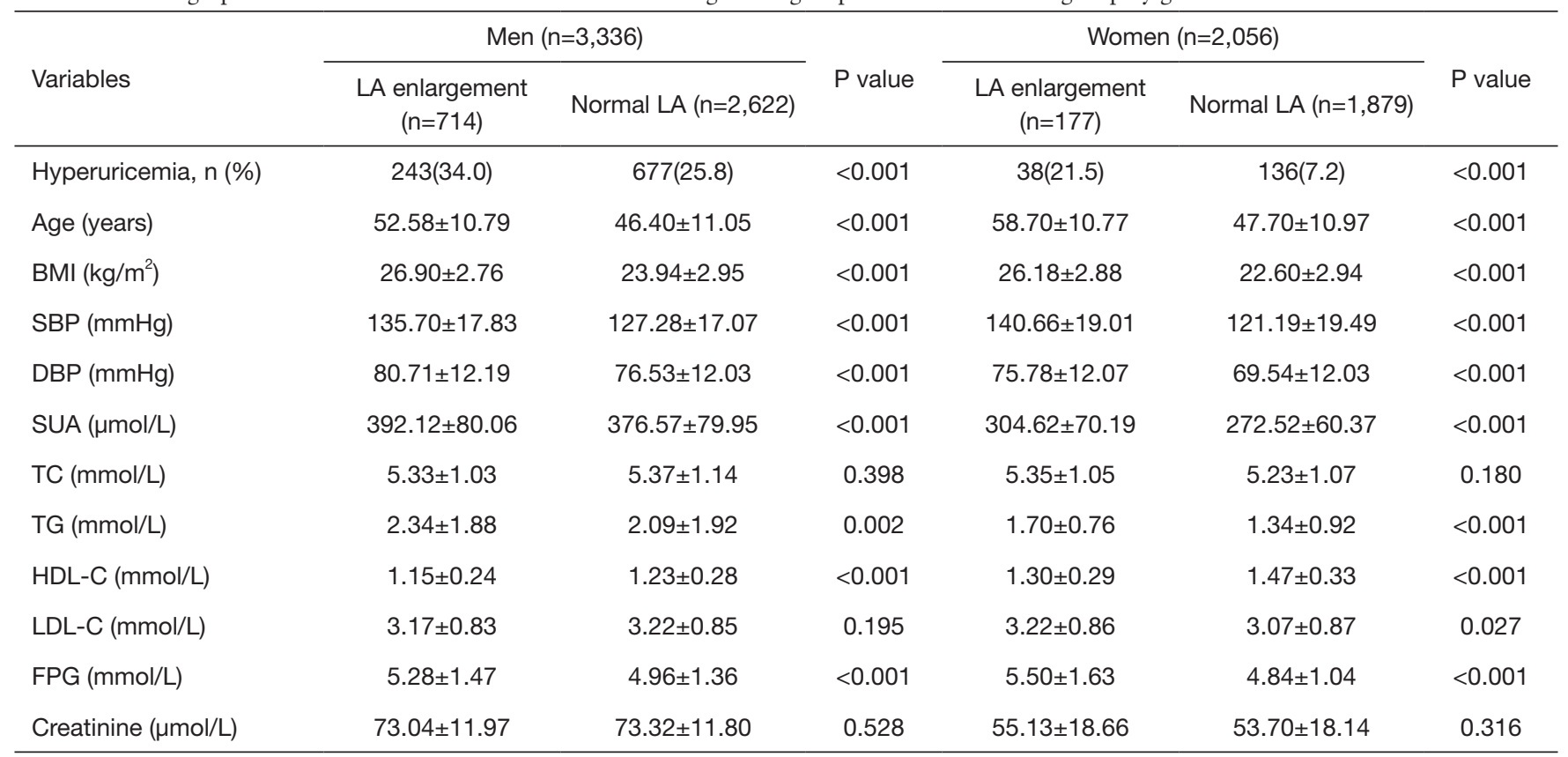

LA, left atrium; BMI, body mass index; SBP, systolic blood pressure; DBP, diastolic blood pressure; SUA, serum uric acid; TC, total cholesterol; TG, triglyceride; HDL-C, high-density lipoprotein cholesterol; LDL-C, low-density lipoprotein cholesterol; FPG, fasting plasma glucose.

Table 3 Univariate and multivariate analyses for LA enlargement in all participants

\begin{tabular}{|c|c|c|c|c|}
\hline Variables & \multicolumn{2}{|c|}{ Univariate analysis } & \multicolumn{2}{|c|}{ Multivariate analysis } \\
\hline Gender, male vs. female & $0.34(0.29-0.41)$ & $<0.001$ & $0.48(0.39-0.59)$ & $<0.001$ \\
\hline Hyperuricemia, yes vs. no & $2.09(1.78-2.45)$ & $<0.001$ & $1.25(1.04-1.51)$ & 0.017 \\
\hline Age (years), $\geq 50$ vs. $<50$ & $2.63(2.27-3.05)$ & $<0.001$ & $2.59(2,18-3.07)$ & $<0.001$ \\
\hline SBP $(\mathrm{mmHg}), \geq 140$ vs. $<140$ & $2.62(2.24-3.05)$ & $<0.001$ & 1.59 (1.33-1.89) & $<0.001$ \\
\hline DBP (mmHg), $\geq 90$ vs. $<90$ & $2.09(1.73-2.52)$ & $<0.001$ & $0.99(0.78-1.26)$ & 0.942 \\
\hline TC (mmol/L) & $1.02(0.95-1.09)$ & 0.612 & - & - \\
\hline $\mathrm{TG}(\mathrm{mmol} / \mathrm{L})$ & $1.14(1.09-1.18)$ & $<0.001$ & $0.94(0.89-0.99)$ & 0.020 \\
\hline FPG (mmol/L) & $1.22(1.17-1.28)$ & $<0.001$ & $1.07(1.02-1.14)$ & 0.013 \\
\hline Creatinine $(\mu \mathrm{mol} / \mathrm{L})$ & $1.019(1.015-1.024)$ & $<0.001$ & $1.00(0.99-1.00)$ & 0.411 \\
\hline
\end{tabular}

OR, odds ratio; Cl, confidence interval; LA, left atrium; BMI, body mass index; SBP, systolic blood pressure; DBP, diastolic blood pressure; TC, total cholesterol; TG, triglyceride; HDL-C, high-density lipoprotein cholesterol; LDL-C, low-density lipoprotein cholesterol; FPG, fasting plasma glucose. 
Table 4 Univariate and multivariate analyses for LA enlargement in women

\begin{tabular}{|c|c|c|c|c|}
\hline Variables & \multicolumn{2}{|c|}{ Univariate analysis } & \multicolumn{2}{|c|}{ Multivariate analysis } \\
\hline Hyperuricemia, yes vs. no & $3.53(2.35-5.26)$ & $<0.001$ & $1.73(1.10 v 2.74)$ & 0.019 \\
\hline Age (years), $\geq 50$ vs. $<50$ & 4.57 (3.20-6.52) & $<0.001$ & $2.59(1.74-3.87)$ & $<0.001$ \\
\hline BMI $\left(\mathrm{kg} / \mathrm{m}^{2}\right), \geq 24$ vs. $<24$ & $4.15(3.31-5.19)$ & $<0.001$ & $3.16(2.47-4.04)$ & $<0.001$ \\
\hline DBP (mmHg), $\geq 90$ vs. $<90$ & $2.24(1.39-3.61)$ & 0.001 & $1.28(0.72-2.27)$ & 0.398 \\
\hline $\mathrm{TC}(\mathrm{mmol} / \mathrm{L})$ & $1.10(0.96-1.27)$ & 0.180 & - & - \\
\hline $\mathrm{TG}(\mathrm{mmol} / \mathrm{L})$ & $1.33(1.18-1.51)$ & $<0.001$ & $0.83(0.68-1.00)$ & 0.056 \\
\hline HDL-C (mmol/L) & $0.17(0.10-0.29)$ & $<0.001$ & $0.28(0.14-0.53)$ & $<0.001$ \\
\hline Creatinine $(\mu \mathrm{mol} / \mathrm{L})$ & $1.01(1.00-1.03)$ & 0.064 & - & - \\
\hline
\end{tabular}

OR, odds ratio; Cl, confidence interval; LA, left atrium; BMI, body mass index; SBP, systolic blood pressure; DBP, diastolic blood pressure; TC, total cholesterol; TG, triglyceride; HDL-C, high-density lipoprotein cholesterol; LDL-C, low-density lipoprotein cholesterol; FPG, fasting plasma glucose.

Table 5 Univariate and multivariate analyses for LA enlargement in men

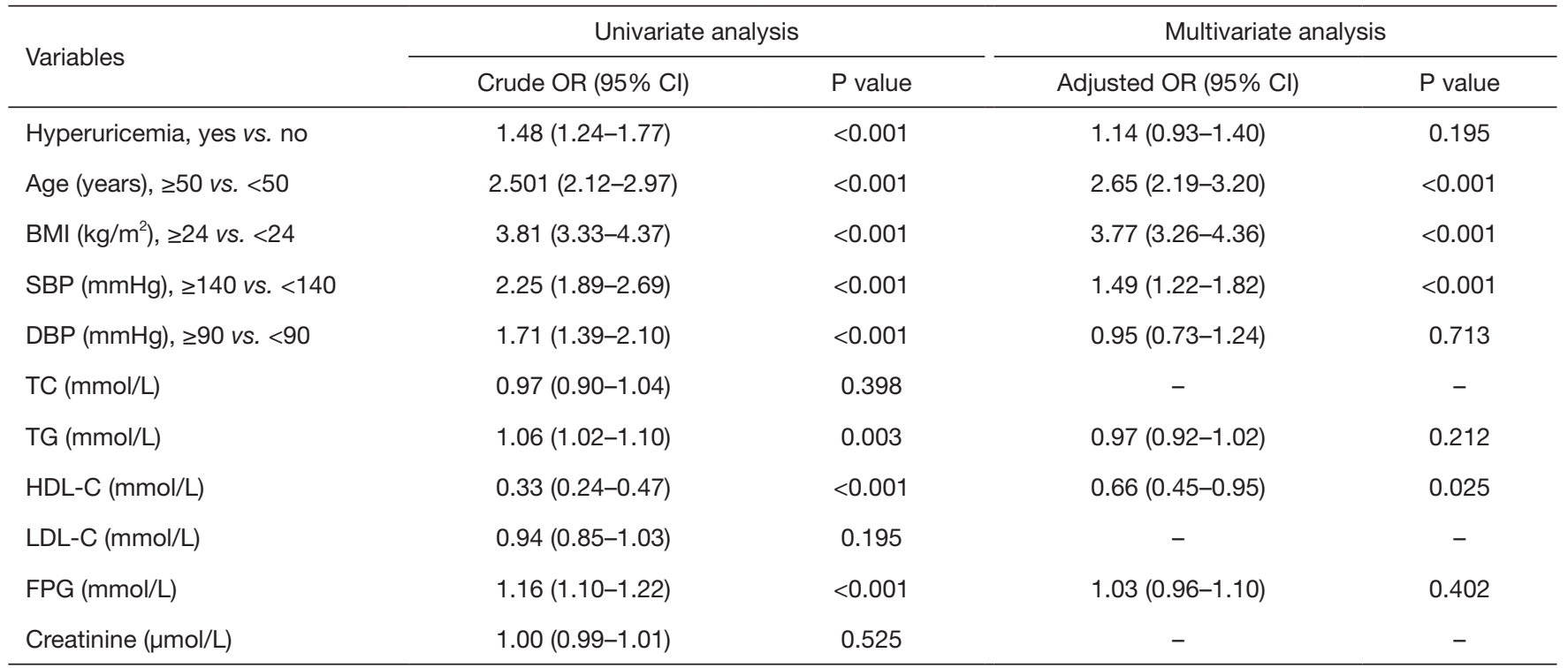

OR, odds ratio; Cl, confidence interval; LA, left atrium; BMI, body mass index; SBP, systolic blood pressure; DBP, diastolic blood pressure; TC, total cholesterol; TG, triglyceride; HDL-C, high-density lipoprotein cholesterol; LDL-C, low-density lipoprotein cholesterol; FPG, fasting plasma glucose. 
with LA enlargement in healthy adults, and after adjustments were made for various clinical and biochemical characteristics, $\mathrm{HU}$ was found to greatly increase the risk of LA enlargement in women. The link between HU and LA has not been demonstrated in studies that use gender as an independent variable before. Thus our study aimed to further investigate the relationship between HU and LA enlargement in different gender in a relatively large cohort.

Recent studies have reported the connection between SUA and LA size. Letsas et al. (8) conducted a study consisting of $86 \mathrm{AF}$ and 48 non-AF patients, and demonstrated that SUA level was significantly related to LA diameter and SUA elevation was a predictor of permanent AF. However, the sample size was very small and was not from a healthy population. Moreover, AF itself may promote atrial remodeling (13), which may interfere with the measurement of the LA diameter. Chao et al. (9) demonstrated that SUA was positively correlated with LA size and HU was associated with a larger LA diameter. Consistent with these results, our study found a significant correlation between HU and LA enlargement in healthy adult participants even after adjusting for various clinical and biochemical characteristics. Therefore, $\mathrm{HU}$ is a significant risk factor for the prevalence of LA enlargement. Structural modeling can lead to LA enlargement, which plays a vital role in the management of $\mathrm{AF}$ (7). Thus, our results revealed atrial remodeling caused by $\mathrm{HU}$ might partially explain the reason why $\mathrm{HU}$ is independently associated with the occurrence of paroxysmal or persistent $\mathrm{AF}(8,9,14-16)$.

The association between HU and AF may possibly be explained by their relationship with inflammation or oxidative stress (2). It is understood that the increased risk of $\mathrm{AF}$ may be related to the behavior of inflammation regulating atrial electrophysiology and structural substrates (17). Inflammation promotes LA enlargement and atrial fibrosis, which can cause AF through atrial conduction disturbance (18). Other studies have reported that the process by which HU causes inflammation is actually through inducing protein expression in cells and activating uric acid transporter (UAT) (19-21). It has been reported that renin-angiotensin-aldosterone system (RAAS) may induce atrial inflammation (22). Some experimental evidence has indicated that SUA may stimulate the circulating and local RAAS in the cardiovascular system $(23,24)$. Xanthine oxidoreductase (XOR) has also been investigated in a number of studies in relation to its critical role in the pathogenesis of AF. A canine model studying left ventricular dysfunction found that xanthine oxidase inhibitors can prevent AF (25). Another study in mice indicated that an increase of SUA can lead to cardiomyocyte hypertrophy and the weakening of myocardial relaxation tension, mainly as a result of an increase in xanthine oxidase activity in heart tissue. These conditions were improved with allopurinol treatment (26). These results may offer an explanation for the relationship between $\mathrm{HU}$ and cardiac structure remodeling. In short, the accumulation of uric acid in cardiomyocytes may lead to ion and structural remodeling of the atria, and UATs can mediate atrial remodeling by regulating the concentration of uric acid in the cells.

The Atherosclerosis Risk in Communities (ARIC) study (27) demonstrated a link between race and gender differences with $\mathrm{HU}$ and $\mathrm{AF}$, with LA enlargement being more obvious among those of Black race or female sex. After adjusting for other covariates, Suzuki et al. (28) found that the level of SUA only affects AF in women; however, the authors could not determine the underlying mechanisms. In this study, the association between $\mathrm{HU}$ and LA enlargement remained independent in women after considering confounding variables, but not in men, suggesting that $\mathrm{HU}$ is specifically associated with an increased risk of LA enlargement in women. Another study found $\mathrm{HU}$ was independently associated with endothelial dysfunction in post-menopausal, but not pre-menopausal women (29). Consistent with the findings of the above study, our results indicated that $\mathrm{HU}$ is independently associated with LA enlargement in women age 50 years or older. This suggests that HU could be an independent risk factor for LA enlargement, particularly in post-menopausal women. Therefore, our results may explain the gender differences in the association of elevated SUA with AF risk. Further study is warranted to explain this connection and explore the potential mechanisms.

Our results showed that in addition to HU, independent variables such as age, BMI, BP, TG, HDL-C, FPG and SCr were related to LA enlargement in total sample size (Table 3). Therefore, in patients age 50 years or older, especially with metabolic syndrome such as obesity, hypertension, hypertriglyceridemia, low HDL-C, and DM, we recommend monitoring and maintaining SUA levels below the upper limit of normal. Specific diets, keep healthy weight and increase physical activity, and uricosuric antihypertensive drugs (losartan) can be used as first choice therapy in these patients; diuretics should be avoided.

Some limitations to our study should be addressed. 
First, due to the cross-sectional design employed, followup research is needed to evaluate the relationships we identified more accurately. Second, this study was single center in nature, and the selection of samples could have been biased towards the Chinese population. Therefore, more prospective clinical trials are needed to verify the influence between HU and LA enlargement in different regions and races. Finally, further prospective studies are needed to determine whether the treatment of HU can improve atrial remodeling and then reduce the occurrence and development of AF.

\section{Conclusions}

Our research shows that $\mathrm{HU}$ is independently connected to LA enlargement in healthy adults and specifically associated with an increased risk of LA enlargement in women.

\section{Acknowledgments}

Funding: This study was supported by grants from the Wenzhou Municipal Sci-Tech Bureau's program (No. Y2020315).

\section{Footnote}

Reporting Checklist: The authors have completed the STROBE reporting checklist. Available at https://dx.doi. org/10.21037/atm-21-3402

Data Sharing Statement: Available at https://dx.doi. org/10.21037/atm-21-3402

Conflicts of Interest: All authors have completed the ICMJE uniform disclosure form (available at https://dx.doi. org/10.21037/atm-21-3402). The authors have no conflicts of interest to declare.

Ethical Statement: The authors are accountable for all aspects of the work in ensuring that questions related to the accuracy or integrity of any part of the work are appropriately investigated and resolved. All procedures performed in this study involving human participants were in accordance with the Declaration of Helsinki (as revised in 2013). Institutional Review Board (IRB) of the first affiliated hospital of Wenzhou Medical University approved the research protocol (No. 2020107) and individual consent for this retrospective analysis was waived.
Open Access Statement: This is an Open Access article distributed in accordance with the Creative Commons Attribution-NonCommercial-NoDerivs 4.0 International License (CC BY-NC-ND 4.0), which permits the noncommercial replication and distribution of the article with the strict proviso that no changes or edits are made and the original work is properly cited (including links to both the formal publication through the relevant DOI and the license). See: https://creativecommons.org/licenses/by-nc-nd/4.0/.

\section{References}

1. Lloyd-Jones D, Adams R, Carnethon M, et al. Heart disease and stroke statistics--2009 update: a report from the American Heart Association Statistics Committee and Stroke Statistics Subcommittee. Circulation 2009;119:e21-181.

2. Maharani N, Kuwabara M, Hisatome I. Hyperuricemia and Atrial Fibrillation. Int Heart J 2016;57:395-9.

3. Iwasaki YK, Nishida K, Kato T, et al. Atrial fibrillation pathophysiology: implications for management. Circulation 2011;124:2264-74.

4. Heijman J, Voigt N, Nattel S, et al. Cellular and molecular electrophysiology of atrial fibrillation initiation, maintenance, and progression. Circ Res 2014;114:1483-99.

5. Bai J, Lu Y, Lo A, Zhao J, Zhang H. PITX2 upregulation increases the risk of chronic atrial fibrillation in a dosedependent manner by modulating $\mathrm{I}(\mathrm{Ks})$ and $\mathrm{I}(\mathrm{CaL})$ -insights from human atrial modelling. Ann Transl Med. 2020 Mar;8:191.

6. Chao TF, Suenari K, Chang SL, et al. Atrial substrate properties and outcome of catheter ablation in patients with paroxysmal atrial fibrillation associated with diabetes mellitus or impaired fasting glucose. Am J Cardiol 2010;106:1615-20.

7. Vaziri SM, Larson MG, Benjamin EJ, et al. Echocardiographic predictors of nonrheumatic atrial fibrillation. The Framingham Heart Study. Circulation 1994;89:724-30.

8. Letsas KP, Korantzopoulos P, Filippatos GS, et al. Uric acid elevation in atrial fibrillation. Hellenic J Cardiol 2010;51:209-13.

9. Chao TF, Hung CL, Chen SJ, et al. The association between hyperuricemia, left atrial size and new-onset atrial fibrillation. Int J Cardiol 2013;168:4027-32.

10. Borghi C, Tykarski A, Widecka K, et al. Expert consensus for the diagnosis and treatment of patient with 
hyperuricemia and high cardiovascular risk. Cardiol J 2018;25:545-63.

11. Leung AA, Nerenberg K, Daskalopoulou SS, et al. Hypertension Canada's 2016 Canadian Hypertension Education Program Guidelines for Blood Pressure Measurement, Diagnosis, Assessment of Risk, Prevention, and Treatment of Hypertension. Can J Cardiol 2016;32:569-88.

12. Lang RM, Bierig M, Devereux RB, et al. Recommendations for chamber quantification: a report from the American Society of Echocardiography's Guidelines and Standards Committee and the Chamber Quantification Writing Group, developed in conjunction with the European Association of Echocardiography, a branch of the European Society of Cardiology. J Am Soc Echocardiogr 2005;18:1440-63.

13. Nattel S, Burstein B, Dobrev D. Atrial remodeling and atrial fibrillation: mechanisms and implications. Circ Arrhythm Electrophysiol 2008;1:62-73.

14. Sun GZ, Guo L, Wang J, et al. Association between hyperuricemia and atrial fibrillation in rural China: a cross-sectional study. BMC Cardiovasc Disord 2015;15:98.

15. Kuwabara M, Niwa K, Nishihara S, et al. Hyperuricemia is an independent competing risk factor for atrial fibrillation. Int J Cardiol 2017;231:137-42.

16. Mantovani A, Rigolon R, Civettini A, et al. Hyperuricemia is associated with an increased prevalence of paroxysmal atrial fibrillation in patients with type 2 diabetes referred for clinically indicated 24-h Holter monitoring. J Endocrinol Invest 2018;41:223-31.

17. Hu YF, Chen YJ, Lin YJ, et al. Inflammation and the pathogenesis of atrial fibrillation. Nat Rev Cardiol 2015;12:230-43.

18. Burstein B, Comtois P, Michael G, et al. Changes in connexin expression and the atrial fibrillation substrate in congestive heart failure. Circ Res 2009;105:1213-22.

19. Kang DH, Park SK, Lee IK, et al. Uric acid-induced C-reactive protein expression: implication on cell proliferation and nitric oxide production of human vascular cells. J Am Soc Nephrol 2005;16:3553-62.

20. Kanellis J, Watanabe S, Li JH, et al. Uric acid stimulates

Cite this article as: Fang D, Wang N, Chen Q, Wu G, Wu J, Zhang W, Fu G. The association between hyperuricemia and left atrial enlargement in healthy adults. Ann Transl Med 2021;9(14):1176. doi: 10.21037/atm-21-3402 monocyte chemoattractant protein-1 production in vascular smooth muscle cells via mitogen-activated protein kinase and cyclooxygenase-2. Hypertension 2003;41:1287-93.

21. Zhang Y, Yamamoto T, Hisatome I, et al. Uric acid induces oxidative stress and growth inhibition by activating adenosine monophosphate-activated protein kinase and extracellular signal-regulated kinase signal pathways in pancreatic $\beta$ cells. Mol Cell Endocrinol 2013;375:89-96.

22. Perlstein TS, Gumieniak O, Hopkins PN, et al. Uric acid and the state of the intrarenal renin-angiotensin system in humans. Kidney Int 2004;66:1465-70.

23. Yu MA, Sánchez-Lozada LG, Johnson RJ, et al. Oxidative stress with an activation of the renin-angiotensin system in human vascular endothelial cells as a novel mechanism of uric acid-induced endothelial dysfunction. J Hypertens 2010;28:1234-42.

24. Corry DB, Eslami P, Yamamoto K, et al. Uric acid stimulates vascular smooth muscle cell proliferation and oxidative stress via the vascular renin-angiotensin system. J Hypertens 2008;26:269-75.

25. Sakabe M, Fujiki A, Sakamoto T, et al. Xanthine oxidase inhibition prevents atrial fibrillation in a canine model of atrial pacing-induced left ventricular dysfunction. J Cardiovasc Electrophysiol 2012;23:1130-5.

26. Jia G, Habibi J, Bostick BP, et al. Uric acid promotes left ventricular diastolic dysfunction in mice fed a Western diet. Hypertension 2015;65:531-9.

27. Tamariz L, Agarwal S, Soliman EZ, et al. Association of serum uric acid with incident atrial fibrillation (from the Atherosclerosis Risk in Communities ARIC study). Am J Cardiol 2011;108:1272-6.

28. Suzuki S, Sagara K, Otsuka T, et al. Gender-specific relationship between serum uric acid level and atrial fibrillation prevalence. Circ J 2012;76:607-11.

29. Maruhashi T, Nakashima A, Soga J, et al. Hyperuricemia is independently associated with endothelial dysfunction in postmenopausal women but not in premenopausal women. BMJ Open 2013;3:e003659.

(English Language Editors: G. Stone and J. Gray) 